\title{
QUANTUM MECHANICAL STUDIES OF BOND AND MOLECULAR POLARIZABILITIES OF GAS-PHASE METAL HALIDES
}

\author{
A.N. PandeY*, A. Bigotto \\ Department of Chemical Sciences, University of Trieste \\ P. le Europa, 34127 Trieste, Italy \\ AND R.K. Gulati \\ International School for Advanced Studies (SISSA) \\ Strada Costiera 11, 34100 Trieste, Italy \\ (Received July 5, 1991; in final version September 11, 1991)
}

The bond and molecular polarizabilities are studied for the gas-phase metal halides using delta-function potential model of chemical binding on the basis of quantum mechanical approach. The applicability of the model is demonstrated. The contribution of the polarity corrections for the metal halides is thoroughly investigated and it has been found that it plays a significant role for fluorides. Our polarizability calculations resolve the discrepancy about the conformation of the barium dichloride and mercury dichloride and favour the nonlinear structure. Due to lack of experimental results for most of halides of transition metals, the decision whether to incorporate polarity corrections or not remains uncertain at this stage: we must await measurements of more experimental values before we reach any final conclusion. The ionic bond orders have been used for the first time to investigate polarizabilities of monomers and dimers of alkali halides. The results for dimers reveal that polarity contribution is essential for the lithium halides dimers and not for other dimers. The computed results will be asset for the interpretation of experimental measurements.

PACS numbers: $31.10 .+z, 31.90 .+\mathrm{s}$

\footnotetext{
*On study leave from the Department of Physics, Meerut College, Meerut, India.
} 


\section{Introduction}

Theoretical studies of the weak interactions between static external or internal fields of the electrons of an atom or molecule can increase our understanding of the electronic structure of a system and aid in the interpretation of experimental measurcments. In this regard the delta-function potential model has played an importanit role in investigating a number of properties related to molecule [1-5]. Lippincott and Stutman [6] extended this model to compute bond and molecular polarizabilities for a variety of molecules. The model has been successfully applied to simple polyatomic molecules having non-polar and polar bonds by Lippincott et al. [7], Nagarajan [8, 9], Sanyal et al. [10] and Pandey et al. [11, 12]. Beran and Kevan [13] have used it to calculate molecular polarizabilities of fluorocarbons, substituted fluorocarbons, ether, esters, ketones and aldehydes as well as a few halogen substituted alkanes and alkyl benzenes. A systematic study of bond and molecular polarizabilities for substituted hydrocarbons with ring and chain structures has been underkarten by Puranchandra and Rammurthy [14], Sanyal et al. [15] and Kumar et al. [16]. Till now a very limited attempt has been made to study the bond and molecular polarizabilities for alkali halides $[17,18]$ possessing ionic bonds and transition metal halides [6].

On account of renaissance in instrumental and theoretical techniques such as spectroscopic methods, electron diffraction, molecular beam deflection, quantum chemical calculations, and combination techniques in past few years more accurate and reliable data on the geometry of the variety of molecules have been accumulated in the literature. Recently, Iargittai [19] has compiled the structural data of gas-phase metal halides, therefore, in the light of current information about the equilibrium geometries of halides, it was thought timely and worthwhile to study the bond and molecular polarizabilities for the gas-phase metal halides using delta-function potential model of chemical binding on quantum mechanical basis with a view to test the applicability of the model for the halides under present investigation on the one hand and present results for the interpretation of experimental measurements, whenever available in the near future, on the other hand.

\section{Polarizability calculations}

The general expression in cartesian coordinates for molecular polarizability is written as:

$$
\alpha=\frac{\frac{2}{3} \sum_{i \neq 0}\left(\mu_{x}\right)_{i}^{2}+\left(\mu_{y}\right)_{i}^{2}+\left(\mu_{z}\right)_{i}^{2}}{E_{i}-E_{0}}
$$

where

$$
\mu_{x i}=\left(\Psi_{i}^{*}\left|\sum_{j} e x_{j}\right| \Psi_{0}\right)
$$

$e$ is the electronic charge, $\Psi_{0}$ is the ground-state delta-function wave function, and $E$ represents the energy. Equation 2 poses difficulty in evaluation, in general, 
except for diatomic molecules where fairly accurate wave functions lead to comparable results with experiments $[17,20]$. In order to compute the polarizabilities for general types of molecules, Lippincott and Stutman [6] used the semiempirical delta-function model of chemical binding $[2,5]$. In this model the colombic potentials in Schrödinger equation of molecular system is replaced by the delta-function potentials. The molecular wave functions are constructed from the linear combinations of atomic delta-function wave function. Using variational procedure [21] first introduced by Hylleraas [22] and Hasse [23] the $x x$-component of the polarizability can be written in the form:

$$
\alpha_{x x}=\frac{4 n A}{a_{0}}\left[\overline{\left(x_{1}-\bar{x}\right)^{2}}-(n-1) \overline{\left(x_{1}-\bar{x}\right)\left(x_{2}-\bar{x}\right)}\right]^{2},
$$

where $x_{1}$ refers to the coordinate of any one of the $n$ equivalence classes of electrons, $\bar{x}$ refers to the average coordinate of any one of these electrons, $A$ is the delta-function strength or reduced electronegativity of the nu us $_{\text {[5] }}$ and $a_{0}$ is the radius of the first Bohr orbit of hydrogen atom. Since the model does not allow any interaction between coordinates, $\left(x_{1}-\bar{x}\right)\left(x_{2}-\bar{x}\right)=0$. Further, with mean delta-function strengths it predicts $\bar{x}=0$, so Eq. (3) takes the form:

$$
\left.\alpha_{x x}=\frac{4 n A}{a_{0}} \frac{{ }^{2}}{\left[x_{1}^{2}\right.}\right]^{2}
$$

or equivalently

$$
\alpha_{x x}=\frac{4 n A}{a_{0}} \sum_{i}{\overline{\left[x_{i}^{2}\right.}}^{2} .
$$

Molecular polarizability is contributing to the parallel and perpendicular components of the constituent bond polarizabilities. The bond parallel component is taken to be the sum of the bonding electron contribution and the contribution from the valence shell non-bonding electrons in each atom of the bond. The bonding electron contribution is calculated by using a linear combination of atomic delta-function wave functions representing the nuclei involved in the bond; i.e. the expectation value of the electron position squared $\bar{x}^{2}$ along the bond axis is calculated and this in turn is used to obtain the bond parallel component of the polarizability $\alpha_{\| b}$ from the equation:

$$
\alpha_{\| b}=4 n A_{12} \frac{{\overline{\left[x^{2}\right]}}^{2}}{a_{0}},
$$

where

$$
\bar{x}^{2}=\frac{R^{2}}{4}+\frac{1}{2 C_{12}^{2}} ; \quad A_{12}=\left(A_{1} A_{2}\right)^{1 / 2},
$$

$n$ is the bond order, $R$ is the internuclear distance at the equilibrium position, and

$$
C_{12}=\left(n_{1} N_{2} N_{1} N_{2}\right)^{1 / 4}\left(A_{1} A_{2}\right)^{1 / 2} \text {. }
$$

Here $n_{i}$ and $N_{i}(i=1,2)$ are the principal quantum number and number of electrons making contributions to the binding, respectively. In case the bond is of 
heteronuclear type, the bond parallel component of the polarizability must be corrected to account for the charge density introduced by the electronegativity difference of the bonding atoms. Here, the charge density in the bond region should then be related to the percent covalent character $\sigma$ believed to exist in the form:

$$
\sigma=\exp \left[-\frac{\left(X_{1}-X_{2}\right)^{2}}{4}\right]
$$

where $X_{1}$ and $X_{2}$ are the electronegativities of the atoms 1 and 2, respectively, on the Pauling's scale [24]. Taking into account the polarity correction, the bond parallel component of the polarizability is given by

$$
\alpha_{\| p}=\sigma \alpha_{\| b} .
$$

The contribution of the non-bond-region electron to the parallel component of the polarizability $\alpha$ is calculated from the fraction of electrons in the valence shell of the given atom not involved in bonding and its atomic polarizability. Lewis-Langmuir octet rule [25, 26] modified by Linnett [27] as a double-quartet of electrons is followed for such calculations. The general expression for the contribution of the non-bond region electrons to the parallel component of the polarizability $\alpha_{\| n}$ is written as:

$$
\alpha_{\| n}=\sum_{j} f_{j} \alpha_{j} .
$$

Here $f_{j}$ is the fraction of the valence electrons in the $j$ th atom not participating in the bonding and $\alpha_{j}$ is the atomic polarizability of the $j$ th atom. Following an empirical approach Lippincott and Stutman [6] obtained a general expression for the evaluation of the perpendicular component of the bond polarizability which is expressed as:

$$
\sum 2 \alpha_{\perp j}=\frac{3 N-2 n_{b}}{N} \sum_{j} \alpha_{j}
$$

where $N$ is the number of atoms and $n_{b}$ is the number of bonds in a molecule. A polarity correction is also applied to the perpendicular bond polarizabilities. The modified expression for the sum of perpendicular components of the bond polarizability is written as:

$$
\sum 2 \alpha_{\perp j}=n_{\mathrm{df}} \sum_{j} \frac{X_{j}^{2} \alpha_{j}}{X_{j}^{2}}
$$

where $n_{\mathrm{df}}=3 N-2 n_{b}$, the residual atomic polarizability degrees of freedom. This is obtained by taking into account the symmetry and geometry of the molecules.

The average molecular polarizability $\alpha_{M}$ without polarity correction can be expressed as:

$$
\alpha_{M}=\frac{1}{3}\left[\sum_{i} \alpha_{i}+\sum_{j} f_{j} \alpha_{j}+\frac{3 N-2 n_{b}}{N} \sum_{j} \alpha_{j}\right]
$$


and with polarity correction:

$$
\alpha_{M}=\frac{1}{3}\left[\sum_{i} \alpha_{i}+\sum_{j} f_{j} \alpha_{j}+\frac{n_{\mathrm{df}} \sum_{j} X_{j}^{2} \alpha_{j}}{\sum_{j} X_{j}^{2}}\right] .
$$

The summation subscript $i$ refers to bonds and $j$ refers to atoms. Equations (14) and (15) are used in the present study to compute average molecular polarizability.

\section{Results and discussion}

The required data for the present computation are the bond lengths, electronegativities on the Pauling's scale [24], delta-function strengths tabulated by Lippincott and Stutman [6], the values of parameter " $c$ ", and the atomic polarizabilities. The bond lengths for most of the halides under present study are taken from the compilation of Hargittai [19] and from literature [28-39] for other halides. The Lewis-Langmuir octet rule [25, 26] modified by Linnett [27] has been followed in accounting for the non-bond contribution in polarizability calculations. In determining the residual atomic polarizability degrees of freedom for the halides, the method suggested by Lippincott and Stutman [6] has been considered. The delta-function strength of an atom in bond of polyatomic halides is calculated using the empirical relation:

$$
A^{* 2}=A^{2}[1-0.20 / n-1],
$$

given by Lippincott and Dayhoff [5], where $A$ is the delta-function strength of an atom in the bond of a diatomic halide, $n$ is the principal quantum number of the atom, and $A^{*}$ is the delta-function strength of an atom in the bond of a polyatomic halide.

The halides, for which the bond parallel components, the contribution of non-bond-region electrons, the bond perpendicular components and the average molecular polarizabilities have been calculated from the delta-function potential model following quantum mechanical approach, are categorized according to their residual atomic polarizability degrees of freedom $n_{\mathrm{df}}$. The results are presented in Tables I to $\mathrm{V}$. The available experimental polarizabilities $[32,33]$ which are determined by measurements of dielectric constant, refractive indices, dipole moments and molar refraction through the well-known Clausius-Mossotti, Langevin-Debye and Lorentz-Lorenz equations are included in Tables I to V.

\subsection{Group 13 and alkali monohalides}

Monohalides of group 13 metals have only single bonds with a double-quartet of electrons around a respective halogen atom. The alkali monohalides have ionic bonds, therefore, in order to account for this effect in our calculations, we have used the ionic bond order [34]. The ionic bond orders [34] were determined from the data presented by DeWijn [35] for alkali monohalides. The computed polarizabilities are summarized in Table I. No experimental values of molecular 
TABLE I

Calculated polarizabilities in $10^{-25} \mathrm{~cm}^{3}$ for group 13 metal and alkali monohalides for four residual atomic polarizability degrees of freedom.

\begin{tabular}{l|r|r|r|r|r}
\hline \hline Halides & $\sum \alpha_{\| p_{i}}$ & \multicolumn{1}{|c|}{$\sum_{\| n}$} & $\sum 2 \alpha_{\perp i}$ & $\alpha_{M}($ calc $)$ & $\alpha_{M}(\exp )$ \\
\hline $\mathrm{AlF}$ & 5.350 & 30.320 & 36.505 & 24.058 & \\
$\mathrm{AlCl}$ & 34.176 & 38.017 & 75.760 & 49.318 & \\
$\mathrm{AlBr}$ & 48.839 & 42.757 & 95.274 & 62.290 & \\
$\mathrm{AlI}$ & 83.224 & 51.594 & 128.896 & 87.905 & \\
$\mathrm{GaF}$ & 7.550 & 41.766 & 47.986 & 32.434 & \\
$\mathrm{GaCl}$ & 39.717 & 49.463 & 93.104 & 60.762 & \\
$\mathrm{GaBr}$ & 54.407 & 54.203 & 114.012 & 74.208 & \\
$\mathrm{GaI}$ & 87.772 & 63.041 & 149.832 & 100.215 & \\
$\mathrm{InF}$ & 12.554 & 56.646 & 64.745 & 44.648 & \\
$\mathrm{InCl}$ & 56.863 & 64.343 & 118.512 & 79.906 & \\
$\mathrm{InBr}$ & 74.506 & 69.083 & 141.484 & 95.024 & \\
$\mathrm{InI}$ & 113.561 & 77.920 & 180.791 & 124.091 & \\
$\mathrm{TlF}$ & 15.061 & 118.319 & 131.606 & 88.329 & \\
$\mathrm{TlCl}$ & 61.261 & 126.061 & 222.073 & 136.450 & \\
$\mathrm{TlBr}$ & 77.836 & 130.756 & 255.162 & 154.584 & \\
$\mathrm{TII}$ & 113.354 & 139.593 & 312.065 & 188.337 & \\
$\mathrm{LiF}$ & 23.118 & 4.200 & 34.901 & 20.740 & $108.00[17]$ \\
$\mathrm{LiCl}$ & 19.915 & 11.897 & 77.940 & 36.584 & $34.60[18]$ \\
$\mathrm{LiBr}$ & 40.061 & 16.637 & 100.500 & 52.400 & \\
$\mathrm{LiI}$ & 49.623 & 25.474 & 104.065 & 59.721 & \\
$\mathrm{NaF}$ & 44.366 & 4.200 & 43.488 & 30.685 & \\
$\mathrm{NaCl}$ & 72.065 & 11.897 & 93.488 & 59.150 & \\
$\mathrm{NaBr}$ & 66.467 & 16.637 & 118.629 & 67.244 & \\
$\mathrm{NaI}$ & 77.425 & 25.474 & 164.369 & 89.089 & \\
$\mathrm{KF}$ & 62.581 & 4.200 & 52.066 & 39.616 & \\
$\mathrm{KCl}$ & 106.093 & 11.897 & 109.176 & 75.955 & \\
$\mathrm{KBr}$ & 117.116 & 16.637 & 136.967 & 90.240 & \\
$\mathrm{KI}$ & 145.940 & 25.474 & 188.067 & 119.827 & \\
& & & & & \\
$\mathrm{RbF}$ & 72.289 & 4.200 & 57.675 & 44.721 & \\
$\mathrm{RbCl}$ & 123.331 & 11.897 & 118.859 & 84.696 & \\
$\mathrm{RbBr}$ & 155.786 & 16.637 & 147.974 & 106.800 & \\
$\mathrm{RbI}$ & 201.684 & 25.474 & 201.614 & 142.924 & \\
$\mathrm{CsF}$ & 76.062 & 4.200 & 61.471 & 47.244 & \\
$\mathrm{CsCl}$ & 134.612 & 11.897 & 126.421 & 90.977 & \\
$\mathrm{CsBr}$ & 174.973 & 16.637 & 157.113 & 115.910 & \\
$\mathrm{CsI}$ & 225.524 & 25.474 & 214.103 & 155.034 & \\
& & & & & \\
& & & & \\
& & \\
& &
\end{tabular}

polarizabilities for the monohalides of group 13 and 1 metals are reported in the literature, but the quantum mechanical results of the polarizability for the monomers of lithium fluoride and lithium chloride exist in literature $[17,18]$. The calculated 
a verage molecular polarizability for lithium chloride is in good agreement with the value reported by Bounds et al. [18] but the agreement is not good in the case of lithium fluoride (see Table I) with the value reported by Kolker and Karplus [17]. Since the magnitude of average molecular polarizability for lithium fluoride should not be greater than the corresponding magnitude for lithium chloride, therefore, the comparison of the two calculated values does not seem justified.

The justification of the use of ionic bond orders in case of alkali monohalides cannot be presented at this stage on account of non-availability of experimental results. However, we have tried to present logical (reasonable) evidence in favour of our approach to utilize ionic bond orders in the computation of polarizabilities for the dimers of the alkali halides possessing ionic bonds in further discussion.

\subsection{Group 2, 14 and 16 dihalides}

The polarizabilities for some dihalides of groups 2, 14 and 16 possessing bent geometries and six residual atomic polarizability degrees of freedom are collected in Table II. The dihalides have only single bonds with a double-quartet of

TABLE II

Calculated and experimental polarizabilities in $10^{-25} \mathrm{~cm}^{3}$ for groups 2, 14 and 16 dihalides of five residual atomic polarizability degrees of freedom.

\begin{tabular}{l|r|r|r|r|r}
\hline \hline Halides & $\sum \alpha_{\| p_{i}}$ & $\sum \alpha_{\| n}$ & $\sum 2 \alpha_{\perp i}$ & $\alpha_{M}$ (calc) & $\alpha_{M}($ exp $)$ \\
\hline $\mathrm{SrCl}_{2}$ & 78.751 & 23.794 & 113.753 & 72.173 & \\
$\mathrm{BaCl}_{2}$ & 84.878 & 23.794 & 119.344 & 76.005 & $79.806[33]$ \\
$\mathrm{BaI}_{2}$ & 200.286 & 50.949 & 214.362 & 155.200 & \\
$\mathrm{SiF}_{2}$ & 13.504 & 23.340 & 35.983 & 24.276 & \\
$\mathrm{SiCl}_{2}$ & 80.648 & 38.734 & 81.603 & 67.000 & \\
$\mathrm{SiBr}_{2}$ & 111.519 & 48.214 & 106.015 & 88.583 & \\
$\mathrm{GeF}_{2}$ & 18.273 & 27.640 & 39.937 & 28.617 & \\
$\mathrm{GeCl}_{2}$ & 93.296 & 43.034 & 88.163 & 74.831 & \\
$\mathrm{GeBr}_{2}$ & 125.523 & 52.514 & 113.378 & 97.138 & \\
$\mathrm{SnCl}_{2}$ & 117.630 & 50.074 & 98.902 & 88.869 & \\
$\mathrm{SnBr}_{2}$ & 159.417 & 59.554 & 125.434 & 114.802 & \\
$\mathrm{SnI}_{2}$ & 234.650 & 77.229 & 172.108 & 161.330 & \\
$\mathrm{PbF}_{2}$ & 28.269 & 76.445 & 84.809 & 63.174 & \\
$\mathrm{PbCl}_{2}$ & 119.030 & 91.839 & 162.611 & 124.494 & $99.46[33]$ \\
$\mathrm{PbBr}_{2}$ & 156.197 & 101.319 & 196.956 & 151.491 & \\
$\mathrm{PbI}_{2}$ & 231.904 & 118.994 & 258.079 & 203.000 & \\
$\mathrm{SeCl}_{2}$ & 123.609 & 40.621 & 83.170 & 82.466 & \\
$\mathrm{TeCl}_{2}$ & 140.393 & 49.141 & 93.152 & 94.229 &
\end{tabular}

electrons around the halogen atoms. As seen from the table, the calculated values of average molecular polarizabilities with polarity corrections for the dichlorides of barium and lead are in reasonable agreement with the experimental values. The 
variation in shape of alkaline earth (group 2) dihalides are reported in literature [19]. Nagarajan [9] computed the average molecular polarizability $\alpha_{M}=84.717 \times$ $10^{-25} \mathrm{~cm}^{3}$ for barium dichloride on the basis of linear structure. As seen from Table II, we have computed this value $\alpha_{M}=76.005 \times 10^{-25} \mathrm{~cm}^{3}$ for it, considering a bent configuration. A comparison of these values with the experimental value $\alpha_{M}=79.806 \times 10^{-25} \mathrm{~cm}^{3}$ for barium dichloride shows that our value is more close to experimental value than the value reported by Nagarajan [9]. Therefore, it is concluded on the basis of our polarizability calculation that the barium dichloride posseses the bent structure. This is further supported by the ionic model in which the bent geometry is favoured by large, more polarizable metal ions and small, more electronegative halide ions [36].

\subsection{Group 2, 12 and first row transition metal dihalides and group 15 trihalides}

Table III includes the results of polarizability calculations for some linear dihalides of groups 2, 12 and first row transition metals and pyramidal trihalides of group 15 elements possessing six residual atomic polarizability degrees of freedom. The dihalides and trihalides have single bonds with a double-quartet of electrons around the halogen atoms. A number of features are evident on examination of results presented in Table III. From the analysis of the available experimental values through refractometric method [33] and the computed results with the polarity correction, it is evident that these are in good agreement. In view of the availability of some new experimental results, we have further computed the polarizabilities without polarity corrections for mercury dichloride and mercury diiodide which are included in Table III. It is remarkable to note the new value for mercury dihalide compares very well with the value calculated without the polarity correction, but it is not so in the case of mercury diiodide. In order to resolve this discrepancy we considered the bent geometry of mercury dichloride and mercury diiodide and again computed the polarizabilities with and without polarity corrections and included the results in Table III. An analysis of these results shows that now the values are more comparable than the previous values with the experimental data for mercury dichloride while in the case of mercury diiodide the comparison becomes poor. It is, therefore, concluded that our results favour the bent structure to mercury dichloride in contrast to linear structure to mercury diiodide. The structural problems are also reported in literature. Spectroscopic results [37-40] have been analysed in terms of the bent structure for several dihalides of mercury on the one hand and indication of linearity for mercury dihalides has also been noticed from molecular beam deflection studies [41] on the other hand. Only one experimental value exists for zinc dichloride and we found no experimental values for the dihalides of first row transition metals, therefore, it is not possible to decide at this stage whether polarity corrections are needed or not in the calculations of molecular polarizabilities for the dihalides of first row transition metals. We, therefore, have calculated the polarizabilities with and without polarity corrections for this group of dihalides and the results are included in Table III. Except for the trifluorides of group 15 elements, where polarity corrections seem essential, we 
TABLE III

Calculated and experimental polarizabilities in $10^{-25} \mathrm{~cm}^{3}$ for groups 2, 12 and first row transition metal dihalides and group 15 trihalides or six residual atomic polarizability degrees of freedom.

\begin{tabular}{|c|c|c|c|c|c|}
\hline Halides & $\overline{\sum \alpha_{\| p_{i}}}$ & $\sum \alpha_{\| n}$ & $\overline{\sum 2 \alpha_{\perp i}}$ & $\overline{\alpha_{M} \text { (calc) }}$ & $\overline{\alpha_{M}(\exp )}$ \\
\hline $\mathrm{MgF}_{2}$ & 8.437 & 8.400 & 49.760 & 22.200 & $18.426[33]$ \\
\hline $\mathrm{MgCl}_{2}$ & 52.806 & 23.794 & 114.311 & 63.636 & 53.851 [33] \\
\hline $\mathrm{CaCl}_{2}$ & 65.456 & 23.794 & 127.768 & 72.400 & \\
\hline $\mathrm{CaBr}_{2}$ & 90.000 & 33.274 & 165.147 & 96.140 & \\
\hline $\mathrm{CaI}_{2}$ & 151.475 & 50.949 & 233.893 & 145.440 & \\
\hline $\mathrm{SrI}_{2}$ & 189.200 & 50.949 & 246.187 & 162.112 & \\
\hline $\mathrm{ZnF}_{2}$ & 13.797 & 8.400 & 56.689 & 26.295 & \\
\hline $\mathrm{ZnCl}_{2}$ & 60.106 & 23.794 & 122.440 & 68.779 & $67.363[33]$ \\
\hline $\mathrm{ZnBr}_{2}$ & 82.354 & 33.274 & 155.946 & 90.525 & \\
\hline $\mathrm{ZnI}_{2}$ & 130.205 & 50.949 & 215.629 & 132.269 & \\
\hline $\mathrm{CdBr}_{2}$ & 119.511 & 33.274 & 165.511 & 106.100 & \\
\hline \multirow{4}{*}{$\mathrm{HgCl}_{2}$} & 95.315 & 23.794 & 175.884 & 98.320 & 90.670 \\
\hline & 95.315 & 23.794 & 146.536 & 88.550 & \\
\hline & 129.000 & 23.794 & 267.980 & 140.250 & \\
\hline & 129.000 & 23.794 & 223.317 & 125.370 & $116.000 \quad[32]$ \\
\hline \multirow[t]{4}{*}{$\mathrm{HgI}_{2}$} & 176.690 & 50.949 & 281.188 & 169.609 & 164.740 [33] \\
\hline & 176.690 & 50.949 & 234.323 & 153.987 & \\
\hline & 193.330 & 50.949 & 331.340 & 191.873 & $191.000 \quad[32]$ \\
\hline & $b^{\prime} \quad 193.330$ & 50.949 & 276.116 & 173.465 & \\
\hline \multirow[t]{2}{*}{$\mathrm{VCl}_{2}$} & 83.574 & 69.748 & 130.130 & 94.484 & \\
\hline & 136.419 & 69.748 & 208.700 & 138.289 & \\
\hline \multirow[t]{2}{*}{$\mathrm{CrF}_{2}$} & 14.720 & 59.455 & 61.262 & 45.146 & \\
\hline & 62.131 & 59.455 & 172.780 & 98.122 & \\
\hline \multirow[t]{2}{*}{$\mathrm{CrCl}_{2}$} & 72.426 & 74.849 & 130.130 & 92.468 & \\
\hline & 118.223 & 74.849 & 208.700 & 133.924 & \\
\hline \multirow[t]{2}{*}{$\mathrm{MnF}_{2}$} & 13.469 & 68.443 & 60.602 & 47.505 & \\
\hline & 64.259 & 68.443 & 187.720 & 106.807 & \\
\hline \multirow[t]{2}{*}{$\mathrm{MnCl}_{2}$} & 68.898 & 83.837 & 130.067 & 94.267 & \\
\hline & 120.920 & 83.837 & 223.640 & 142.800 & \\
\hline \multirow[t]{2}{*}{$\mathrm{MnBr}_{2}$} & 94.005 & 93.317 & 165.137 & 117.486 & \\
\hline & 143.430 & 93.317 & 245.760 & 160.836 & \\
\hline \multirow[t]{2}{*}{$\mathrm{FeF}_{2}$} & 17.644 & 72.837 & 74.092 & 54.860 & \\
\hline & 59.169 & 72.837 & 191.432 & 107.813 & \\
\hline \multirow[t]{2}{*}{$\mathrm{FeCl}_{2}$} & 77.754 & 88.231 & 149.211 & 105.065 & \\
\hline & 111.447 & 88.231 & 227.352 & 142.343 & \\
\hline \multirow[t]{2}{*}{$\mathrm{FeBr}_{2}$} & 106.555 & 97.711 & 184.794 & 129.687 & \\
\hline & 136.819 & 97.711 & 249.472 & 161.334 & \\
\hline $\mathrm{PF}_{3}$ & 26.519 & 22.068 & 38.876 & 29.154 & \\
\hline \multirow[t]{2}{*}{$\mathrm{PCl}_{3}$} & 134.239 & 45.159 & 91.527 & 90.310 & $103.030[33]$ \\
\hline & 164.371 & 45.159 & 97.965 & 102.498 & $128.000[32]$ \\
\hline
\end{tabular}




\begin{tabular}{|c|c|c|c|c|c|}
\hline \multicolumn{6}{|c|}{ TABLE III (cont.) } \\
\hline$\overline{\text { Halides }}$ & $\overline{\sum \alpha_{\| p_{i}}}$ & $\sum \alpha_{\| n}$ & $\sum 2 \alpha_{\perp i}$ & $\alpha_{M}($ calc $)$ & $\alpha_{M}(\exp )$ \\
\hline \multirow[t]{2}{*}{$\mathrm{PBr}_{3}$} & 187.243 & 59.379 & 120.496 & 122.372 & \\
\hline & 211.644 & 59.379 & 122.850 & 131.291 & \\
\hline \multirow[t]{2}{*}{$\mathrm{PI}_{3}$} & 324.070 & 85.891 & 171.408 & 193.790 & \\
\hline & 337.296 & 85.891 & 169.245 & 197.477 & \\
\hline $\mathrm{AsF}_{3}$ & 32.851 & 25.808 & 42.378 & 33.679 & \\
\hline \multirow{2}{*}{$\mathrm{AsCl}_{3}$} & 153.986 & 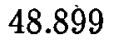 & 98.098 & 100.325 & \\
\hline & 197.722 & 48.899 & 112.000 & 119.540 & $149.000[32]$ \\
\hline \multirow[t]{2}{*}{$\mathrm{AsBr}_{3}$} & 207.968 & 63.119 & 128.329 & 133.139 & \\
\hline & 244.053 & 63.119 & 136.875 & 148.016 & \\
\hline \multirow[t]{2}{*}{$\mathrm{AsI}_{3}$} & 320.376 & 89.631 & 181.801 & 197.269 & \\
\hline & 341.039 & 89.631 & 183.270 & 204.647 & \\
\hline $\mathrm{SbF}_{3}$ & 55.152 & 32.056 & 47.757 & 45.000 & \\
\hline \multirow[t]{2}{*}{$\mathrm{SbCl}_{3}$} & 185.276 & 55.147 & 107.876 & 116.100 & \\
\hline & 250.723 & 55.147 & 135.420 & 147.097 & \\
\hline \multirow[t]{2}{*}{$\mathrm{SbBr}_{3}$} & 245.044 & 69.367 & 139.796 & 151.403 & \\
\hline & 300.047 & 69.367 & 160.305 & 176.573 & \\
\hline \multirow[t]{2}{*}{$\mathrm{SbI}_{3}$} & 375.113 & 95.579 & 196.648 & 222.447 & \\
\hline & 410.439 & 95.579 & 206.700 & 237.573 & \\
\hline \multirow[t]{2}{*}{$\mathrm{BiCl}_{3}$} & 185.929 & 84.391 & 159.610 & 143.310 & \\
\hline & 251.606 & 84.391 & 245.085 & 193.694 & \\
\hline \multirow[t]{2}{*}{$\mathrm{BiBr}_{3}$} & 262.470 & 98.611 & 198.166 & 186.416 & \\
\hline & 321.385 & 98.611 & 269.970 & 230.000 & \\
\hline \multicolumn{6}{|c|}{1 - linear structure with polarity corrections } \\
\hline \multicolumn{6}{|c|}{ b - bent structure with polarity corrections } \\
\hline \multicolumn{6}{|c|}{$\begin{array}{l}\mathrm{l}^{\prime}-\text { linear structure without polarity corrections } \\
\mathrm{b}^{\prime}-\text { bent structure without polarity corrections }\end{array}$} \\
\hline \multicolumn{6}{|c|}{$\mathrm{b}^{\prime}$ - bent structure without polarity corrections } \\
\hline
\end{tabular}

have computed the polarizabilities for the remaining trihalides of this group with and without polarity corrections and results are included in Table III. It is interesting to contrast the polarizabilities calculated with and without polarity corrections.

From the survey of the computed results it has been found that there is an indication that the values calculated without polarity corrections are relatively more comparable to the values calculated with polarity corrections. It is true that the difference between the values calculated with and without polarity corrections increases in trihalides of this group keeping the ligand constant with the decrease of electronegativity of the central atom, but the difference decreases keeping the central atom constant with the decrease of the electronegativity of the halogen atom. However, the polarity-corrected values for the trihalides of group 15 elements show poorer agreement with the available experimental values than do the polarizabilities calculated without polarity corrections. Thus the decision to make use of the polarity correction or not to calculate molecular polarizabilities is rather a delicate one which cannot be decided on the ground of chemical arguments alone. We conclude that for trihalides of group 15 elements except for trifluorides, the 
value calculated without polarity corrections can give comparable results with the experimental values.

\subsection{Group 13 and first row transition metal trihalides and group 14 transition metal tetrahalides}

The results calculated with and without polarity corrections for the halides possessing seven residual atomic polarizability degrees of freedom are tabulated

Calculated and experimental polarizabilities in $10^{-25} \mathrm{~cm}^{3}$ for group 13 and first row transition metal trihalides and group 14 and transition metal tetrahalides of seven residual atomic polarizability degrees of freedom.

\begin{tabular}{|c|c|c|c|c|c|}
\hline Halides & $\sum \alpha_{\| p_{i}}$ & $\sum \alpha_{\| n}$ & $\sum 2 \alpha_{\perp i}$ & $\overline{\alpha_{M}(\text { calc })}$ & $\overline{\alpha_{M}(\exp )}$ \\
\hline \multirow{2}{*}{$\mathrm{BCl}_{3}$} & 73.229 & 35.691 & 96.889 & 68.603 & $80.05[33]$ \\
\hline & 94.028 & 35.691 & 96.635 & 75.451 & $93.80[32]$ \\
\hline \multirow[t]{2}{*}{$\mathrm{AlF}_{3}$} & 15.250 & 12.600 & 45.044 & 24.044 & \\
\hline & 72.752 & 12.600 & 94.290 & 59.881 & \\
\hline \multirow[t]{2}{*}{$\mathrm{AlCl}_{3}$} & 91.219 & 35.691 & 110.783 & 79.231 & $89.435 \quad[33]$ \\
\hline & 160.096 & 35.691 & 141.435 & 112.407 & \\
\hline \multirow[t]{2}{*}{$\mathrm{AlBr}_{3}$} & 137.920 & 49.911 & 147.953 & 111.928 & 124.464 [33] \\
\hline & 210.433 & 49.911 & 170.467 & 143.604 & \\
\hline \multirow[t]{2}{*}{$\mathrm{AlI}_{3}$} & 266.845 & 76.423 & 215.135 & 186.134 & $199.079 \quad[33]$ \\
\hline & 342.636 & 76.423 & 224.595 & 214.551 & \\
\hline \multirow[t]{2}{*}{$\mathrm{GaF}_{3}$} & 20.267 & 12.600 & 52.535 & 28.467 & \\
\hline & 85.540 & 12.600 & 124.337 & 74.159 & \\
\hline \multirow[t]{2}{*}{$\mathrm{GaI}_{3}$} & 219.060 & 76.423 & 230.434 & 175.305 & \\
\hline & 265.229 & 76.423 & 254.642 & 198.765 & \\
\hline \multirow[t]{2}{*}{$\mathrm{InI}_{3}$} & 145.348 & 76.423 & 253.804 & 158.524 & \\
\hline & 255.852 & 76.423 & 293.702 & 208.659 & \\
\hline \multirow{2}{*}{$\mathrm{ScF}_{3}$} & 16.487 & 12.600 & 58.031 & 29.040 & \\
\hline & 102.012 & 12.600 & 208.740 & 107.784 & \\
\hline \multirow[t]{2}{*}{$\mathrm{VF}_{3}$} & 20.353 & 43.236 & 59.709 & 41.100 & \\
\hline & 85.906 & 43.236 & 159.757 & 96.300 & \\
\hline \multirow[t]{2}{*}{$\mathrm{CrF}_{3}$} & 19.421 & 50.895 & 59.709 & 43.342 & \\
\hline & 81.971 & 50.895 & 159.757 & 97.541 & \\
\hline \multirow[t]{2}{*}{$\mathrm{FeF}_{3}$} & 26.089 & 66.297 & 70.160 & 54.182 & \\
\hline & 87.490 & 66.297 & 176.078 & 109.955 & \\
\hline \multirow[t]{2}{*}{$\mathrm{SiF}_{4}$} & 24.650 & 16.800 & 42.526 & 28.059 & 33.010 [33] \\
\hline & 82.664 & 16.800 & 69.272 & 56.245 & 54.500 \\
\hline \multirow{2}{*}{$\mathrm{SiCl}_{4}$} & 140.917 & 47.590 & 106.408 & 98.304 & 111.72 \\
\hline & 201.980 & 47.590 & 119.560 & 123.042 & \\
\hline \multirow[t]{2}{*}{$\mathrm{SiBr}_{4}$} & 186.594 & 66.548 & 142.733 & 131.958 & $156.140 \quad[33]$ \\
\hline & 239.592 & 66.548 & 150.528 & 152.223 & \\
\hline
\end{tabular}


TABLE IV - (cont.)

\begin{tabular}{|c|c|c|c|c|c|}
\hline Halides & $\overline{\sum \alpha_{\| p_{i}}}$ & $\sum \alpha_{\| n}$ & $\overline{\sum 2 \alpha_{\perp i}}$ & $\alpha_{M}($ calc $)$ & $\alpha_{M}(\exp )$ \\
\hline \multirow{2}{*}{$\mathrm{SiI}_{4}$} & 331.931 & 101.897 & 208.169 & 214.000 & \\
\hline & 375.188 & 101.897 & 208.264 & 228.450 & \\
\hline \multirow[t]{2}{*}{$\mathrm{GeF}_{4}$} & 31.648 & 16.800 & 45.626 & 31.358 & $36.460[33]$ \\
\hline & 106.130 & 16.800 & 81.312 & 68.081 & \\
\hline \multirow[t]{2}{*}{$\mathrm{GeCl}_{4}$} & 162.967 & 47.590 & 111.378 & 107.312 & $121.230[33]$ \\
\hline & 233.584 & 47.590 & 131.600 & 137.599 & $151.000[32]$ \\
\hline \multirow[t]{2}{*}{$\mathrm{GeBr}_{4}$} & 224.458 & 66.548 & 148.370 & 146.460 & \\
\hline & 288.209 & 66.548 & 162.568 & 172.449 & \\
\hline \multirow[t]{2}{*}{$\mathrm{GeI}_{4}$} & 359.239 & 101.897 & 215.075 & 225.404 & \\
\hline & 406.056 & 101.897 & 220.304 & 242.752 & \\
\hline \multirow[t]{2}{*}{$\mathrm{SnCl}_{4}$} & 210.375 & 47.590 & 119.516 & 125.827 & $137.700[33]$ \\
\hline & 301.537 & 47.590 & 151.312 & 166.813 & $180.000[32]$ \\
\hline \multirow[t]{2}{*}{$\mathrm{SnBr}_{4}$} & 284.041 & 66.548 & 157.600 & 169.396 & $189.070[33]$ \\
\hline & 364.715 & 66.548 & 182.280 & 204.514 & $220.000[32]$ \\
\hline \multirow[t]{2}{*}{$\mathrm{SnI}_{4}$} & 425.336 & 101.897 & 226.383 & 251.205 & $277.810[33]$ \\
\hline & 480.764 & 101.897 & 240.016 & 274.226 & $323.000[32]$ \\
\hline $\mathrm{TiF}_{4}$ & 24.023 & 16.800 & 53.119 & 31.314 & \\
\hline \multirow[t]{2}{*}{$\mathrm{TiCl}_{4}$} & 130.684 & 47.590 & 126.058 & 101.444 & 141.000 [33] \\
\hline & 229.358 & 47.590 & 195.412 & 157.453 & $164.000 \quad[32]$ \\
\hline \multirow[t]{2}{*}{$\mathrm{TiBr}_{4}$} & 187.275 & 66.548 & 166.166 & 140.000 & \\
\hline & 285.739 & 66.548 & 226.380 & 192.889 & \\
\hline \multirow[t]{2}{*}{$\mathrm{TiI}_{4}$} & 299.864 & 101.897 & 239.448 & 213.736 & \\
\hline & 385.034 & 101.897 & 284.116 & 257.015 & \\
\hline \multirow[t]{2}{*}{$\mathrm{ZrF}_{4}$} & 27.751 & 16.800 & 56.086 & 33.546 & \\
\hline & 150.395 & 16.800 & 180.936 & 116.044 & \\
\hline \multirow[t]{2}{*}{$\mathrm{ZrCl}_{4}$} & 153.068 & 47.590 & 131.771 & 110.810 & \\
\hline & 290.290 & 47.590 & 231.122 & 189.701 & \\
\hline \multirow[t]{2}{*}{$\mathrm{ZrBr}_{4}$} & 206.434 & 66.548 & 173.024 & 148.669 & \\
\hline & 336.966 & 66.548 & 262.192 & 221.902 & \\
\hline \multirow[t]{2}{*}{$\mathrm{ZrI}_{4}$} & 356.607 & 101.897 & 248.711 & 235.738 & \\
\hline & 439.272 & 101.897 & 319.928 & 287.032 & \\
\hline \multirow[t]{2}{*}{$\mathrm{IrrF}_{4}$} & 22.371 & 16.800 & 70.373 & 36.481 & \\
\hline & 138.418 & 16.800 & 313.950 & 156.389 & \\
\hline \multirow[t]{2}{*}{$\mathrm{IIfCl}_{4}$} & 125.166 & 47.590 & 157.038 & 109.931 & \\
\hline & 257.789 & 47.590 & 364.238 & 223.205 & \\
\hline \multirow[t]{2}{*}{$\mathrm{HfBr}_{4}$} & 169.831 & 66.548 & 202.175 & 146.185 & \\
\hline & 298.063 & 66.548 & 395.206 & 253.272 & \\
\hline \multirow[t]{2}{*}{$\mathrm{Hft}_{4}$} & 278.787 & 101.897 & 285.897 & 222.086 & \\
\hline & 399.594 & 101.897 & 452.942 & 318.144 & \\
\hline \multirow[t]{2}{*}{$\mathrm{VCl}_{4}$} & 100.544 & 62.907 & 126.303 & 107.756 & \\
\hline & 218.826 & 62.907 & 184.954 & 155.562 & \\
\hline \multirow[t]{2}{*}{$\mathrm{VBr}_{4}$} & 181.068 & 81.867 & 166.078 & 143.000 & \\
\hline & 259.530 & 81.867 & 215.922 & 185.773 & \\
\hline
\end{tabular}


in Table IV. It includes the planar trihalides of group 13 elements as monomers, planar trifluorides of first row transition metals, tetrahalides of group 14 and transition metals. Trihalides and tetrahalides have single bond with a double-quartet of electrons around the halogen atoms. A close examination of the results (Table IV) for the trihalides of group 13 elements shows that though the two sets of calculated values are comparable with the available experimental values in the case of trihalides of aluminium but the values calculated with polarity corrections are relatively more close to the experimental values calculated without polarity corrections. In contrast to this the value calculated without polarity corrections for boron trichloride is more close to the experimental value. It is, therefore, concluded that in case of trihalides of aluminium the introduction of polarity corrections in polarizability calculations seems useful. Due to lack of experimental values for the trifluorides of first row transition metals, the decision whether to incorporate polarity corrections or not remains uncertain, however our results on fluorides and the results reported by others $[6,13]$ are in favour of inclusion of polarity corrections for calculating polarizability for fluorides by employing delta-function potential model. A survey of the results for tetrahalides of the group 15 shows that for tetrafluorides the polarity corrections are necessary to obtain satisfactory agreement with the experimental values. For tetrachlorides in general both sets of values are in reasonable agreement with the experimental values while the polarizabilities calculated with no polarity corrections for the tetrabromides and tetraiodides are in reasonable agreement with the experimental values. Only one experimental value exists for the polarizability for titanium tetrachloride and we found no experimental data for other tetrahalides of transition metals. It is seen from Table IV that the available experimental value for titanium tetrachloride is in reasonable agreement with the value calculated without polarity corrections. At present it is not possible to decide about the incorporation of polarity corrections in the polarizability calculations with the model employed here, however, we must await the measurements of more experimental values before we reach to any final conclusion. For tetrafluorides the polarity correction is advised as discussed above.

\subsection{Group 15 and 5 pentahalides, dimers of group 13 tetrahalides and alkali halides}

Table $\mathrm{V}$ includes the results of calculations for the monomeric pentahalides of groups 15, 5 and 6 elements, dimers group 13, trihalides and alkali halide dimers possessing eight residual atomic polarizability degrees of freedom.

\subsubsection{Monomers}

The monomeric pentahalides of the group 15 and group 5 have regular trigonal bibyramidal configuration [19] and pentafluoride of chromium (group 6) has a lower $C_{2 v}$ symmetry than the more symmetric $D_{3 h}$ structure due to dynamic Jahn-Teller effect [42]. On the basis of the structure of pentahalides there is the double-quartet of electrons around the peripheral halogen atoms while ten electrons surround the central arsenic, antimony, vanadium, niobium and tantalum atoms. The group 6 transition metals also have pentahalides in the present case 
e.g. chromium pentafluoride where the electronic configuration is $d^{1}$ and it is degenerate with the one electron occupying $e^{\prime \prime}$ orbital in a considered $D_{3 h}$ symmetry structure. Though the pentahalides partly obey Lewis-Langmuir octet rule $[25,26]$ and Linnett model [27], the agreement between the experimental and calculated values (Table V) of the a verage molecular polarizability is good for antimony pentachloride. Since the experimental and calculated values with polarity corrections are in good agreement, we have only included the polarizabilities calculated by incorporating polarity corrections for all pentahalides.

TABLE V

Calculated and experimental polarizabilities in $10^{-25} \mathrm{~cm}^{3}$ for group 15 and 5 pentahalides and dimers of group 13 trihalides and alkali halides of eight-residual atomic polarizability degrees of freedom.

\begin{tabular}{l|r|r|r|r|r}
\hline \hline Halides & $\sum \alpha_{\| p_{i}}$ & $\sum \alpha_{\| n}$ & $\sum 2 \alpha_{\perp i}$ & $\alpha_{M}($ calc $)$ & $\alpha_{M}(\exp )$ \\
\hline $\mathrm{AsF}_{5}$ & 50.883 & 21.000 & 64.358 & 45.413 & \\
$\mathrm{SbCl}_{5}$ & 292.300 & 59.485 & 131.691 & 161.159 & $156.758[33]$ \\
$\mathrm{VF}_{5}$ & 31.301 & 21.000 & 56.981 & 36.427 & \\
$\mathrm{NbCl}_{5}$ & 211.759 & 59.485 & 143.732 & 138.325 & \\
$\mathrm{TaCl}_{5}$ & 180.387 & 59.485 & 168.522 & 136.131 & \\
$\mathrm{TaBr}_{5}$ & 247.083 & 83.185 & 218.404 & 182.891 & \\
$\mathrm{CrF}_{5}$ & 30.634 & 33.760 & 56.984 & 40.459 & \\
$\mathrm{Al}_{2} \mathrm{~F}_{6}$ & 43.700 & 22.400 & 51.479 & 39.193 & \\
$\mathrm{Al}_{2} \mathrm{Cl}_{6}$ & 292.105 & 63.451 & 126.609 & 160.722 & $158.183[9]^{*}$ \\
$\mathrm{Al}_{2} \mathrm{Br}_{6}$ & 412.659 & 88.731 & 169.578 & 223.656 & \\
$\mathrm{Al}_{2} \mathrm{I}_{6}$ & 678.324 & 135.863 & 245.868 & 353.352 & \\
$\mathrm{Ga}_{2} \mathrm{Cl}_{6}$ & 321.604 & 63.451 & 140.464 & 175.173 & $182.024[9]^{*}$ \\
$\mathrm{Ga}_{2} \mathrm{Br} 6$ & 443.863 & 88.731 & 184.288 & 238.961 & \\
$\mathrm{Ga}_{2} \mathrm{I}_{6}$ & 630.674 & 135.353 & 263.353 & 343.297 & \\
$\mathrm{Fe}_{2} \mathrm{Cl} l_{6}$ & 362.058 & 170.827 & 172.785 & 235.223 & \\
$\mathrm{AlFeCl}_{6}$ & 325.847 & 117.149 & 150.079 & 197.692 & \\
$\mathrm{Tl}_{2} \mathrm{~F}_{2}$ & 87.676 & 8.400 & 263.211 & 119.762 & \\
$(\mathrm{LiF})_{2}$ & 139.582 & 8.400 & 69.802 & 72.595 & $70.000[32]$ \\
$(\mathrm{LiCl})_{2}$ & 203.458 & 23.794 & 155.880 & 127.711 & $130.000[32]$ \\
$(\mathrm{LiBr})_{2}$ & 218.626 & 33.274 & 201.000 & 151.000 & $190.000[32]$ \\
$(\mathrm{LiI})_{2}$ & 251.267 & 50.949 & 282.130 & 194.782 & $230.000[32]$ \\
$(\mathrm{NaCl})_{2}$ & 328.825 & 23.794 & 285.440 & 212.686 & $230.000[32]$ \\
$(\mathrm{NaBr})_{2}$ & 362.248 & 33.274 & 296.500 & 230.674 & $270.000[32]$ \\
$(\mathrm{KCl})_{2}$ & 635.764 & 23.794 & 459.620 & 373.059 & $320.000[32]$ \\
$(\mathrm{KBr})_{2}$ & 772.079 & 33.274 & 470.680 & 425.344 & $420.000[32]$ \\
$(\mathrm{RbCl})_{2}$ & 666.659 & 23.794 & 532.540 & 407.664 & $430.000[32]$ \\
$(\mathrm{RbBr})_{2}$ & 845.264 & 33.274 & 543.600 & 474.050 & $480.000[32]$ \\
$(\mathrm{CsCl})_{2}$ & 624.104 & 23.794 & 742.100 & 463.333 & $420.000[32]$ \\
$(\mathrm{CsBr})_{2}$ & 986.748 & 33.274 & 753.160 & 591.061 & $540.000[32]$ \\
${ }^{*} \mathrm{calculated} \mathrm{value}$ & & & & \\
& & & & & \\
& & &
\end{tabular}




\subsubsection{Dimers}

There are two types of dimers structure. One is the dimeric group 13 halides and iron trichloride and another is the alkali halide dimers. Both types of dimers have eight residual atomic polarizability degrees of freedom. According to spectroscopic studies $[43,44]$ the dimers of the group 13 trihalides belong to $D_{2 h}$ point group, this implies a planar four-membered ring. The ground state electronic configuration for the trihalide dimer satisfies the Linnett model [27]. The electron diffraction studies on iron trichloride and thallous fluoride dimers $[45,46]$ are in favour of a planar rhombic structure and also possess the $D_{2 h}$ point group and four-memebered ring but for the thallous fluoride dimer other structural models are presented in literature [19]. The polarizabilities calculated including polarity corrections are listed in Table V. Due to non-availability of experimental data for the first type of dimers, we have compared our results with those reported by Nagarajan [9] which are included in Table V. It is evident that our values for the dimers of the trichlorides of aluminium and gallium are in good agreement with the values reported by Nagarajan [9]. It seems, therefore, important to use the polarity correction in the calculations of polarizabilities using delta-function potential model for the type of dimers of group 13 trihalides as in the case of monohalides of this group (Table I), however, to arrive at a definite conclusion about the configuration of thallous fluoride dimers with the aid of computed data we must wait till experimental measurements are made. Alkali halides dimers possess a planar rhombic structure with $D_{2 h}$ symmetry [47-49] containing four-membered ring similar to the dimers discussed above. The dimers have ionic bond, therefore, to take into account this effect in our calculation a similar procedure has been followed as discussed for the monomers. Since the measurements of molecular polarizabilities of the alkali halide dimers using molecular beam deflection technique [50] are now available in the literature [32], therefore, it is possible at this stage to examine the justification of the approach followed in computing the polarizabilities for molecules possessing ionic bonds and to take decision of whether to incorporate polarity corrections or not for the alkali halide dimers. First we computed the polarizabilities for all alkali halides dimers under present study taking into account the polarity correction. On comparing the computed results with the experimental results it was found that except for the dimers of lithium halides in other alkali halide dimers the computed results were too low, therefore, we recalculated the polarizabilities without polarity corrections for the last dimers. The results are included in Table V. Examination of the results displayed in this table shows that the calculated values are in good agreement except the lithium bromide dimer where the error is about $21 \%$. On the basis of reasonable agreement between computed and experimental values, in general, we conclude that the use of ionic bond orders for alkali halides and its dimers is justified and the polarity correction is essential for the lithium halide dimers while for other alkali halide dimers the calculation without polarity corrections give reasonable results. 


\section{Conclusion}

To conclude, we will emphasize the following point. After all, the delta-function potential model extended by Lippincott and Stutman for computing molecular polarizabilities gives reasonable good results for gas-phase metal halides. The polarity corrections are necessary for fluorides but in the case of halides for which no experimental data exist at present we must await for measurements of experimental values for them to decide whether to include polarity corrections or not in such calculations. For alkali halides possessing ionic bonds the ionic bond order can be used successfully to compute molecular polarizabilities under present approach. For transition metal halides there is an indication to exclude the polarity correction in such calculations, but a firm confirmation requires more experimental results. The application of this method to complex metal halides is in progress.

\section{Acknowledgements}

One of the authors (ANP) is grateful to the Third World Academy of Sciences (ICTP), Trieste, Italy for awarding him the research grant. He is thanful to the authorities of Meerut College, Meerut, India for granting him study leave. He is obliged to Prof. G. De Alti, Director of the department, for providing him the necessary facilities and cordial atmosphere. He is also indebted to Swedish Agency for Research Cooperation (SEREC) for sponsoring his current Associateship visit to the ICTP Trieste.

\section{References}

[1] K. Ruedenberg, R.G. Parr, J. Chem. Phys. 19, 1268 (1951); 21, 1565 (1953).

[2] A.A. Frost, J. Chem Phys. 22, 1613 (1954); 23, 985 (1955); 25, 1150 (1956).

[3] A.A. Frost, F.E. Leland, J. Chem. Phys. 25, 1154 (1956).

[4] E.R. Lippincott, J. Chem. Phys. 23, 603 (1953); 26, 1678 (1957).

[5] E.R. Lippincott, M.O. Dayhoff, Spectrochim. Acta 16, 807 (1960).

[6] E.R. Lippincott, J.M. Stutman, J. Phys. Chem. 68, 2926 (1964).

[7] E.R. Lippincott, G. Nagarajan, J.M. Stutman, J. Phys. Chem. 70, 78 (1966).

[8] G. Nagarajan, Z. Nat.forsch. A 21, 864 (1966).

[9] G. Nagarajan, Acta Phys. Pol. A43, 301 (1973).

[10] N.K. Sanyal, L. Dixit, A.N. Pandey, Indian J. Pure Appl. Phys. 10, 329 (1972).

[11] A.N. Pandey, D.K. Sharma, S.L. Gupta, V. Kumar, K. Balasubramanyan, Indian J. Phys. B 51, 251 (1977).

[12] J.R. Chopra, A.N. Pandey, U.P. Verma, B. Strauch, Acta Phys. Pol. A65, 351 (1984).

[13] J.A. Beran, L. Kevan, J. Phys. Chem. 73, 3860 (1969). 
[14] B. Puranchandra Rao, V. Ramamurthy, Cur. Sci. 41, 15 (1972).

[15] N.K. Sanyal, P. Parvez, L. Dixit, J. Phys. Chem. 77, 2552 (1973).

[16] V. Kumar, U.P. Verma, A.N. Pandey, Chem. Phys. Lett. 56, 571 (1978).

[17] H.J. Kolker, M. Karplus, J. Chem. Phys. 39, 2011 (1963).

[18] D.G. Bounds, J.H.R. Clarke, A. Hinchliffe, Chem. Phys. Lett. 45, 367 (1977).

[19] M. Hargittai, Coord. Chem. Rev. 91, 35 (1988).

[20] R.M. Stevens, R.M. Pitzer, W.N. Lipscomb, J. Chem Phys. 38, 550 (1963); R.M. Stevens, W.N. Lipscomb, ibid. 40, 2238 (1964).

[21] J.O. Hirschfelder, C.F. Curtiss, B.B. Bird, Molecular Theory of Gases and Liquids, Wiley, New York 1954, p. 942.

[22] E.A. Hylleraas, Z. Phys. 65, 209 (1930).

[23] H.R. Hasse, Proc. Cambridge Phil. Soc. 26, 542 (1930); 27, 66 (1931).

[24] L. Pauling, The Nature of Chemical Bonds, Cornell Univ. Press., Ithaca, New York 1960.

[25] G.N. Lewis, J. Am. Chem. Soc. 38, 762 (1916).

[26] I. Langmuir, J. Am. Chem. Soc. 38, 2221 (1916).

[27] J.W. Linnett, J. Am. Chem. Soc. 83, 2643 (1961).

[28] K.H. Hellwege, A.M. Hellwege (Eds), Landolt Börnstein Numerical Data and Fundamental Relationships in Science and Technology New Series, Springer-Verlag, Vol. 7, Berlin 1976 and Vol. 15, Berlin 1987.

[29] L. Nalbandian, G.N. Papatheodorou, B.N. Cyvin, J. Brenvoll, S.J. Cyvin, Spectrosc. Lett. 22, 1 (1989).

[30] S.J. Cyvin, A. Phongsatha, Spectrosc. Lett. 8, 405 (1975).

[31] A. Phongsatha, S.J. Cyvin, Spectrosc. Lelt. 7, 365 (1974).

[32] Handbook of Chemistry and Physics, CRC Press Boca Raton, 69th edition, Florida 1988-1989, p. E68.

[33] S.S. Batsnov, Refractometry and Chemical Structure, Consultants Bureau, New York 1961.

[34] R.S. Mulliken, J. Chem. Phys. 32, 1841 (1955).

[35] H.W. DeWijn, J. Chem. Phys. 44, 810 (1966).

[36] M.C. Drake, G.M. Rosenblatt, J. Electrochem. Soc. 126, 1387 (1979).

[37] A. Loewenschuss, A. Givan, Ber. Bunsenges. Phys. Chem. 82, 69 (1978).

[38] A. Loewenschuss, A. Ron, O. Schnepp, J. Chem. Phys. 49, 272 (1968); 50, 2502 (1969).

[39] A. Givan, A. Loewenschuss, J. Chem. Phys. 65, 1851 (1976).

[40] A. Givan, A. Loewenschuss, J. Mol. Struct. 48, 325 (1978).

[41] A. Buchler, J.L. Stauffer, W. Klemperer, J. Chem. Phys. 40, 3471 (1964).

[42] I.R. Beattie, J.S. Ogden, R.S. Wyatt, J. Chem. Soc. Dalton Trans. 2343 (1983).

[43] T. Tomita, C.E. Sjogren, P. Klaeboe, G.N. Papatheodorou, E. Rytter, J. Raman Spectrosc. 14, 415 (1983).

[44] C.E. Sjogren, P. Klaeboe, E. Rytter, Spectrochim. Acta A 40, 457 (1984).

[45] M. Hargittai, J. Tremmel, I. Hargittai, J. Chem. Soc. Dalton Trans. 87 (1980). 
[46] V.G. Solomonik, E.Z. Zasorin, G.V. Girichev, K.S. Krasnov, Izv. Vyssh. Uchebn. Zaved. Khim. Tekhnol. 17, 136 (1974).

[47] V.G. Solomonik, K.S. Krasnov, G.V. Girichev, E.Z. Zasorin, Zh. Strukt. Khim. 20, 427 (1979).

[48] R.J. Mawhorter, M. Fink, J.G. Hartley, J. Chem. Phys. 83, 4418 (1985).

[49] T.P. Martin, H. Schaber, J. Chem. Phys. 68, 4299 (1978); 70, 2029 (1979).

[50] R. Kremens, B. Bederson, B. Jaduszliwer, J. Stockdale, A. Tino, J. Chem. Phys. 81, 1676 (1984). 